\title{
Probing Universality in AdS/CFT
}

\author{
Adam Ritz
}

University of Victoria

\author{
with P. Kovtun [0801.2785, 0806.0110] \\ and J. Ward [0811.4195]
}

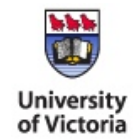

Shifmania workshop - Minneapolis - May 2009 


\section{Happy Birthday \\ Misha!}

Oops...some fine print:

- this talk contains no strong gauge dynamics.........just the weakly-coupled dual - this talk crosses no (phase) boundaries.........just sticks to the critical endpoint! 


\section{Happy Birthday}

Misha!

Oops...some fine print:

- this talk contains no strong gauge dynamics.........just the weakly-coupled dual

- this talk crosses no (phase) boundaries..........just sticks to the critical endpoint! 


\section{Are all fixed points alike?}

Which $d$-dimensional fixed points behave like classical GR in $\mathrm{AdS}_{d+1}$ ?

For example, when is $\mathrm{AdS}_{4} / \mathrm{CFT}_{3}$ most applicable to phenomena in condensed matter physics [Herzog et al. '07, Hartnoll et al. '07,...]

- There are strongly-interacting near-critical regimes for which AdS/CFT may provide a novel (and unique) toolkit

- Reverse engineering may allow CM physics to unlock some generic aspects of gravity, and the AdS/CFT correspondence 


\section{Static Universality}

\section{Static universality class criteria}

- spatial dimension

- symmetry of the order paramater
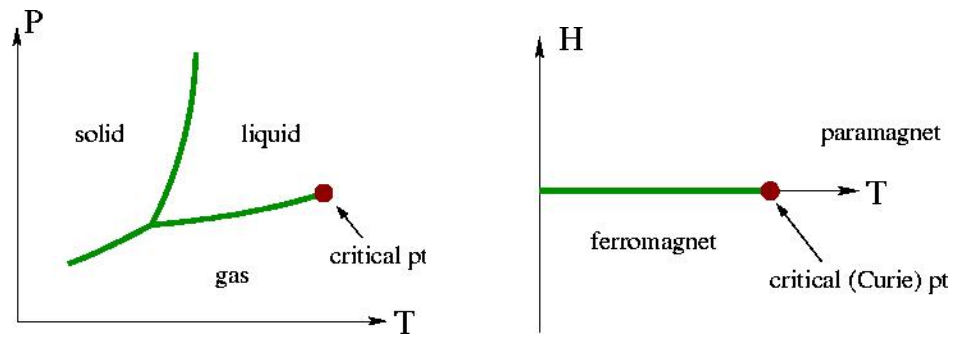

Both critical points are described by the same Ising CFT in $d=3$. 


\section{Dynamic Universality}

\section{Dynamic universality class criteria [Hohenberg \& Halperin '77]}

- spatial dimension

- symmetry of the order parameter

- conserved currents, ...
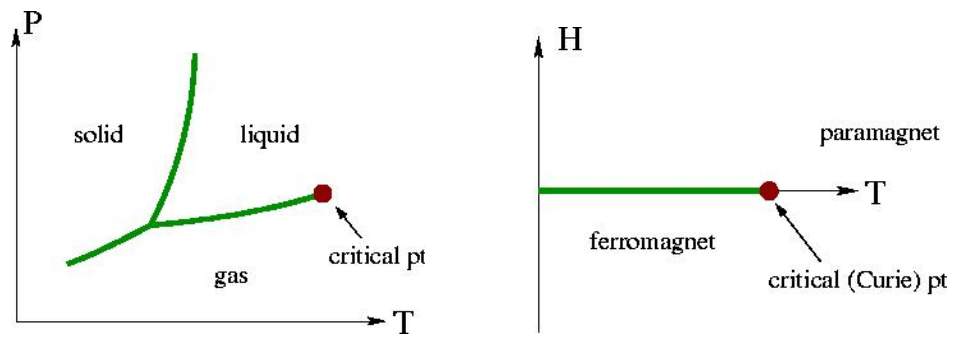

The dynamic universality classes of these critical points are different! 


\section{Quantum Critical Universality}

When are these notions of universality "the same"?

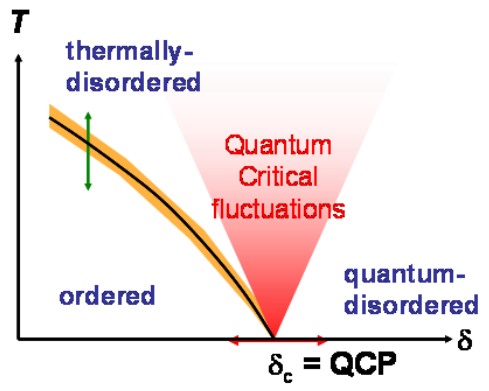




\section{Quantum Critical Universality}

When are these notions of universality "the same"?

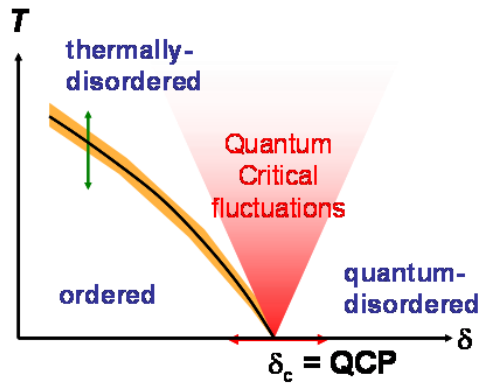

CFTs with classical AdS gravity duals comprise a special subset of this class with correlated vacuum, thermodynamic and (hydro)dynamic properties

NB: this implies taking a single dominant scale (temperature) 


\section{Outline}

(1) Thermodynamics from central charges

(2) Hydrodynamics from central charges

(3) Conclusions 


\section{Outline}

(1) Thermodynamics from central charges

(2) Hydrodynamics from central charges 


\section{Vacuum CFTs and central charges}

For a CFT vacuum state, symmetry determines the correlators of conserved currents:

$$
\left\langle T_{\mu \nu}(x) T_{\alpha \beta}(0)\right\rangle=\frac{c}{x^{2 d}} \Pi_{\mu \nu \alpha \beta}
$$

- $c$ - "measures" the total degrees of freedom

$$
\left\langle J_{\mu}(x) J_{\nu}(0)\right\rangle=\frac{k}{x^{2(d-1)}} \Pi_{\mu \nu}
$$

- $k$ - "measures" the charged degrees of freedom 


\section{Thermal CFTs}

For a CFT, with temperature the only scale:

$$
p(T)=\left.\frac{T}{V} \ln \operatorname{Tr}\left[e^{-\beta(H-\mu Q)}\right]\right|_{\mu \rightarrow 0}=c^{\prime} T^{d}+\frac{1}{2} k^{\prime} \mu^{2} T^{d-2}+\cdots
$$

- $c^{\prime}$ measures entropy density $s=c^{\prime} d T^{d-1}$

- $k^{\prime}$ measures charge susceptibility $\chi=k^{\prime} T^{d-2}$ 


\section{Claim}

- In $d=2$, conformal symmetry relates the vacuum and thermal states [Bloete et al. '86, Affleck et al. '86]:

$$
d=2 \quad \longrightarrow \quad c^{\prime}=\frac{\pi}{6} c \quad k^{\prime}=\frac{1}{2 \pi} k
$$

so thermodynamics is uniquely fixed by the central charges.

- In $d>2$, conformal symmetry does not imply this constraint

BUT: it is true in general for CFTs with gravity duals

$$
\begin{array}{llll}
d=3 & \longrightarrow & c^{\prime}=\frac{\pi^{3}}{162} c & k^{\prime}=\frac{\pi}{24} k \\
d=4 & \longrightarrow & c^{\prime}=\frac{\pi^{2}}{80} c & k^{\prime}=\frac{1}{12} k
\end{array}
$$




\section{AdS gravity dual}

The dual bulk geometry describing the CFT state, and the fluctuations which determine correlators of the conserved currents, follow at leading order in EFT from the Einstein-Maxwell action:

$S=\frac{1}{16 \pi G_{d+1}} \int \mathrm{d}^{d+1} x \sqrt{-g}\left[R+\frac{d(d-1)}{L^{2}}\right]-\frac{1}{4 g_{d+1}^{2}} \int \mathrm{d}^{d+1} x \sqrt{-g} F^{2}+\cdots$

where $G_{d+1}$ and $g_{d+1}^{2}$ encode details of the CFT.

$$
\text { Vacuum state } \leftrightarrow \operatorname{AdS}_{d+1}: d s^{2}=z^{-2}\left(d z^{2}+d \mathbf{x}^{2}\right)
$$

Large volume thermal state $\leftrightarrow \mathrm{RNAdS}_{d+1} \mathrm{BH}: T=T_{\mathrm{BH}}, \mu=\left.A_{t}\right|_{z \rightarrow 0}$ 


\section{Central charges and thermodynamics}

- Perturbing the vacuum state, we obtain the correlators:

$$
\begin{array}{rlll}
\left\langle T_{x y}\left(x_{1}\right) T_{x y}\left(x_{2}\right)\right\rangle & \sim \frac{\partial^{2} S^{\text {on-shell }}}{\partial h^{x y}\left(x_{1}\right) h^{x y}\left(x_{2}\right)} \quad \Longrightarrow \quad c=\operatorname{const}(d) \times \frac{L^{d-1}}{G_{d+1}} \\
\left\langle J_{x}\left(x_{1}\right) J_{x}\left(x_{2}\right)\right\rangle & \sim \frac{\partial^{2} S^{\text {on }- \text { shell }}}{\partial A^{x}\left(x_{1}\right) A^{x}\left(x_{2}\right)} \quad \Longrightarrow \quad k=\operatorname{const}(d) \times \frac{L^{d-3}}{g_{d+1}^{2}}
\end{array}
$$

- From the thermal state dual to the black hole (with $\mu \rightarrow 0$ ):

$$
\begin{array}{ccc}
s=\frac{A_{D-2}}{4 G_{d+1} V}=c^{\prime} d T^{d-1} & \Longrightarrow & c^{\prime}=\operatorname{const}(d) \frac{L^{d-1}}{G_{d+1}} \\
\left.\chi\right|_{\mu=0}=\frac{\rho}{\mu}=k^{\prime} T^{d-2} & \Longrightarrow & k^{\prime}=\operatorname{const}(d) \frac{L^{d-3}}{g_{d+1}^{2}}
\end{array}
$$




\section{Thermodynamics from central charges}

Central charges $(c, k)$ determine $(s, \chi)$ in CFTs with AdS gravity duals

$$
\begin{aligned}
\frac{c^{\prime}}{c} & =\frac{1}{4 \pi^{d / 2}}\left(\frac{4 \pi}{d}\right)^{d} \frac{\Gamma(d / 2)^{3}}{\Gamma(d)} \frac{(d-1)}{d(d+1)} \\
\frac{k^{\prime}}{k} & =\frac{1}{2 \pi^{d / 2}}\left(\frac{4 \pi}{d}\right)^{d-2} \frac{\Gamma(d / 2)^{3}}{\Gamma(d)}
\end{aligned}
$$

In the most relevant cases of $d=3,4$ :

$$
\begin{aligned}
d=3 & \longrightarrow & c^{\prime}=\frac{\pi^{3}}{162} c & k^{\prime} & =\frac{\pi}{24} k \\
d=4 & \longrightarrow & c^{\prime}=\frac{\pi^{2}}{80} c & k^{\prime} & =\frac{1}{12} k
\end{aligned}
$$




\section{Outline}

\section{(1) Thermodynamics from central charges}

(2) Hydrodynamics from central charges 


\section{Hydrodynamics}

Assuming hydrodynamics controls the IR dynamics near the critical point:

$$
\partial_{\mu} T^{\mu \nu}=\partial_{\mu} J^{\mu}=0,
$$

where (for a conformal fluid)

$$
\begin{aligned}
T^{\mu \nu} & =\frac{\epsilon}{3}\left(4 u^{\mu} u^{\nu}+\eta^{\mu \nu}\right)-2 \eta \partial_{\langle\mu} u_{\nu\rangle}+\cdots \\
J^{\mu} & =\rho u^{\mu}-D \Pi_{\nu}^{\mu} \partial_{\nu} \rho+\cdots
\end{aligned}
$$

- $D=\sigma / \chi$ is the diffusion const

- $\eta$ is the shear viscosity

[NB: Strictly we require $J_{\mu} \rightarrow e J_{\mu}$ so $\sigma$ and $\chi$ are implicitly proportional to $e^{2}$ ] 


\section{Transport from thermodynamics}

Using Kubo relations, and the AdS/CFT prescription [Policastro et al '02]:

$$
\begin{gathered}
\eta=-\lim _{\omega \rightarrow 0} \operatorname{Im} \frac{1}{\omega}\left\langle T_{x y} T_{x y}\right\rangle(\omega, \mathbf{0})_{\mathrm{R}}=\frac{1}{16 \pi G_{d+1}}\left(\frac{4 \pi L}{d}\right)^{d-1} T^{d-1} \\
\sigma=-\lim _{\omega \rightarrow 0} \operatorname{Im} \frac{1}{\omega}\left\langle J_{x} J_{x}\right\rangle(\omega, \mathbf{0})_{\mathrm{R}}=\frac{1}{g_{d+1}^{2}}\left(\frac{4 \pi L}{d}\right)^{d-3} T^{d-3} .
\end{gathered}
$$

It follows that CFTs with gravity duals have transport coefficients determined by thermodynamics!

$$
\frac{\eta}{s}=\frac{1}{4 \pi}, \quad \frac{\sigma}{\chi}=D=\frac{1}{4 \pi T} \frac{d}{d-2} .
$$




\section{Transport from central charges}

Putting the pieces together [Kovtun \& AR '08] :

$$
\begin{gathered}
\sigma=\frac{\chi}{4 \pi T} \frac{d}{d-2}=\left[\frac{1}{8 \pi^{d / 2+1}} \frac{d}{d-2}\left(\frac{4 \pi}{d}\right)^{d-2} \frac{\Gamma(d / 2)^{3}}{\Gamma(d)}\right] k T^{d-3} . \\
\eta=\frac{s}{4 \pi}=\left[\frac{1}{16 \pi^{d / 2+1}} \frac{d-1}{d+1}\left(\frac{4 \pi}{d}\right)^{d} \frac{\Gamma(d / 2)^{3}}{\Gamma(d)}\right] c T^{d-1} .
\end{gathered}
$$

General relations, independent of field content, symmetries, etc!

$\chi(\omega)$

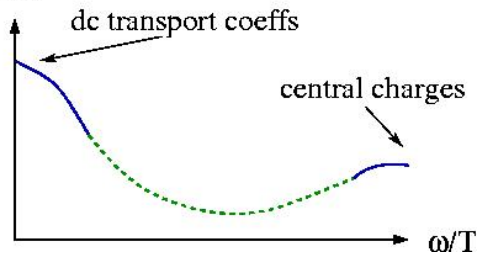

The two limits $\omega / T \rightarrow 0$ and $\omega / T \rightarrow \infty$ are generally determined by different physics [Sachdev '99] 


\section{Central charges and symmetry breaking}

A corrollary (cf. weak gavity conjecture [Arkani-Hamed et al '06])

$$
\frac{\eta}{\sigma}=\left[\frac{8 \pi^{2}(d-1)(d-2)}{d^{3}(d+1)}\right] \frac{c}{k} T^{2} \propto \frac{g_{d+1}^{2}}{G_{d+1}}
$$

The ratio $c / k$ also determines when the $U(1)$ symmetry is unstable to condensation forming a superfluid (or superconducting) phase. In $d=3$, the instability occurs when [Denef \& Hartnoll '09]:

$$
q^{2} \geqslant(3+2 \Delta(\Delta-3)) \frac{k}{c}
$$

with $q(\Delta)$ the charge (dimension) of the operator breaking $\mathrm{U}(1)$. 


\section{Comparisons}

- $d=4$ : The AdS value is possible if $2 n_{s}+n_{f}=8 n_{v}$, and in general:

$$
\frac{3}{8} \leqslant \frac{c /\left.c^{\prime}\right|_{\text {free }}}{c /\left.c^{\prime}\right|_{\text {AdS }}} \leqslant \frac{9}{4} .
$$

- $d=3$ : In this case $\left(c / c^{\prime}\right)^{\text {free }} \propto 1 / \zeta(3)$ so the AdS value is not possible at weak coupling.

For the $\mathrm{O}(N)$ model at large $N$ [Sachdev '93, Chubukov et al '94]

$$
\frac{c^{\prime} /\left.c\right|_{\mathrm{O}(\mathrm{N})}}{c^{\prime} /\left.c\right|_{\mathrm{AdS}}} \approx 1.07, \quad \frac{k^{\prime} /\left.k\right|_{\mathrm{O}(\mathrm{N})}}{k^{\prime} /\left.k\right|_{\mathrm{AdS}}} \approx 1.31, \quad \text { BUT } \quad \sigma /\left.\chi\right|_{\mathrm{O}(\mathrm{N})} \sim \mathcal{O}(N) .
$$

[Cf. The proposed higher-spin AdS dual [Klebanov \& Polyakov '02]] 


\section{Outline}

(1) Thermodynamics from central charges

(2) Hydrodynamics from central charges

(3) Conclusions 


\section{Conclusions}

- CFTs with AdS (gravity) duals are a precise subset with linked vacuum, thermodynamic, and hydrodynamic properties

- Are there physical quantum critical regimes with these features?

Bulk higher dimension operators generically correct these universal relations:

- $R^{n>1}$ terms correct $\eta / s$ [Buchel et al '04; Brigante et al '07, Kats and Petrov '07]

- $R F^{2}$ corrects $\sigma / \chi$ [AR \& Ward '08]

- Can symmetries protect these relations beyond the classical gravity (large $N, \lambda$ ) limit? Are some regimes in the swampland?

Are there other universal relations of this type, associated with perturbing by other "control" paramaters, or for NR quantum critical pts?

- finite chemical potential or magnetic field (and zero T) - extremal BHs

- the confinement or $\chi \mathrm{SB}$ scales? 